\title{
Therapeutic bronchoscopy in the era of genotype directed lung cancer management
}

\author{
Arjun Mohan ${ }^{1}$, Kassem Harris ${ }^{2}$, Mark R. Bowling ${ }^{1}$, Craig Brown ${ }^{1}$, Wolfgang Hohenforst-Schmidt ${ }^{3}$ \\ ${ }^{1}$ Division of Pulmonary, Critical Care and Sleep Medicine, Department of Medicine, East Carolina University-Brody School of Medicine, Greenville, \\ North Carolina, USA; ${ }^{2}$ Interventional Pulmonology Section, Pulmonary Critical Care and Sleep division, Department of Medicine, Westchester \\ Medical Center, Valhalla, New York, USA; ${ }^{3}$ Sana Clinic Group Franken, Department of Cardiology/Pulmonology/Intensive Care/Nephrology, "Hof” \\ Clinics, University of Erlangen, Hof, Germany \\ Contributions: (I) Conception and design: K Harris, W Hohenforst-Schmidt, A Mohan; (II) Administrative support: None; (III) Provision of study \\ materials or patients: All authors; (IV) Collection and assembly of data: All authors; (V) Data analysis and interpretation: All authors; (VI) Manuscript \\ writing: All authors; (VII) Final approval of manuscript: All authors. \\ Correspondence to: Arjun Mohan, MD. Division of Pulmonary, Critical Care and Sleep Medicine, Department of Medicine, East Carolina University- \\ Brody School of Medicine, 3E-149, 600 Moye Boulevard, Greenville, North Carolina 27834, USA. Email: mohana14@ecu.edu.
}

\begin{abstract}
Lung cancer is the leading cause of cancer related deaths. Non-small cell lung cancer (NSCLC) accounts for $\sim 85 \%$ of lung cancers. Our understanding of driver mutations and genotype directed therapy has revolutionized the management of advanced NSCLC. Commonly described mutations include mutations in epidermal growth factor $(E G F R) \& B R A F$ and translocations in anaplastic lymphoma kinase $(A L K) \&$ rat osteosarcoma (ROS1). Drugs directed against these translocations have significantly improved progression free survival individually and have shown a survival benefit when studied in the Lung Cancer Mutation Consortium (median survival 3.5 vs. 2.4 years compared to standard therapy). In a related yet parallel universe, the number of bronchoscopic ablative modalities available for management of cancer related airway obstruction have increased exponentially over the past decade. A wealth of literature has given us a better understanding of the technical aspects, benefits and risks associated with these procedures. While they all show benefits in terms of relieving airway obstruction, symptom control, quality of life and lung function testing, their complication rates vary based on the modality. The overall complication rate was $~ 4 \%$ in the AQuIRE registry. Bronchoscopic therapeutic modalities include rigid bronchoscopy with mechanical debulking, laser, thermo-coagulation [electrocautery \& argon plasma coagulation (APC)], cryotherapy, endobronchial brachytherapy (EBT), photodynamic therapy (PDT), intratumoral chemotherapy (ITC) and transbronchial needle injection (TBNI) of chemotherapy. Intuitively, one would assume that the science of driver mutations would crisscross with the science of bronchoscopic ablation as they overlap in the same patient population. Sadly, this is not the case and there is a paucity of literature looking at these fields together. This results in several unanswered questions about the interplay between these two therapies.
\end{abstract}

Keywords: Lung neoplasm; bronchoscopy; ablation techniques

Submitted Jul 23, 2018. Accepted for publication Aug 03, 2018.

doi: $10.21037 /$ jtd.2018.08.14

View this article at: http://dx.doi.org/10.21037/jtd.2018.08.14

\section{Introduction}

Lung cancer is the leading cause of cancer related deaths in men and women (1) and is responsible for 1.6 million deaths per year worldwide (2). Non-small cell lung cancer
(NSCLC) accounts for $85 \%$ of lung cancers. Over the past decade, there has been a lot of emphasis on understanding driver mutations in NSCLC. Molecular testing for these mutations has advanced our understanding of NSCLC and resulted in genotype directed targeted therapy. Driver 
mutations with approved targeted therapies include epidermal growth factor $(E G F R)$ mutation, anaplastic lymphoma kinase $(A L K)$ translocation, rat osteosarcoma (ROS1) translocation and BRAF mutation. Other mutations being actively investigated include human epidermal growth factor receptor 2 (HER2) mutation, mesenchymal epithelial transition (MET) abnormalities, rearranged during transfection (RET) translocation, RAS mutation and PIK3CA, AKT1 \& phosphatase and tensin homolog (PTEN) alterations.

Proximal or central airway obstruction (CAO) complicates $20-30 \%$ of lung cancer cases (3) and is responsible for $40 \%$ of deaths (4). CAO can occur from varying mechanisms including direct luminal compromise, airway edema/inflammation, and destruction of airway cartilage. There are multiple bronchoscopic ablative techniques available for management of CAO. Common modalities include rigid bronchoscopy with mechanical debulking, laser, thermo-coagulation [electrocautery \& argon plasma coagulation (APC)], cryotherapy, endobronchial brachytherapy (EBT), photodynamic therapy (PDT), intratumoral chemotherapy (ITC) and transbronchial needle injection (TBNI) of chemotherapy. Selection of the appropriate modality depends on several factors including features of the tumor, the patient's clinical presentation, and operator preferences.

Intuitively, the patients who would most often benefit from genotype directed therapy i.e., those with advanced NSCLC, would also be the ones who are most likely to need bronchoscopic ablative therapy. It would be important to understand the influence of driver mutations on outcomes of these procedures. For example, we need to know if certain mutations result in tumors which have higher complication rates with a given procedure. This would influence procedure selection and even the selection of a multi-modality approach. More importantly, we would also like to know if certain bronchoscopic procedure complement certain therapies. For example, will certain driver mutations have a better outcome if we were to combine the gene directed therapy for it with one procedure as opposed to another. Unfortunately, there is very little literature in this field. The scope of this review is to consolidate our current knowledge of immunologic markers of cancer and bronchoscopic ablative modalities. We searched PubMed with the following search terms: bronchoscopy/ablation techniques and lung neoplasms. We limited our search to the past 10 years (given the more recent focus on cancer immunology), English language and human based publications. Articles that focused on diagnostic bronchoscopic techniques were excluded or limited.

\section{Understanding immunotherapy in lung cancer}

Driver mutations (Table 1) occur in cancer cells within genes responsible for cell growth and survival. They are found in about $64 \%$ of patients with NSCLC (26). Identification of some of these mutations, most notably EGFR mutation, $A L K-1$ translocation and ROS-1 translocation has led to a paradigm shift in cancer therapy since the early 2000 s. Along with extent of disease, squamous $v s$. non-squamous history and programmed death ligand (PD-L1) expression, driver mutations heavily influence the choice of therapy in advanced NSCLC. Molecular testing for these driver mutations is mostly done by polymerase chain reaction (PCR), fluorescence in situ hybridization (FISH), nextgeneration sequencing (NGS) and immunohistochemical (IHC) analysis. Another increasingly popular molecular diagnostic tool is liquid biopsy (which is beyond the scope of this paper). The Lung Cancer Mutation Consortium published data in 2014 that showed a survival benefit (median survival 3.5 vs. 2.4 years) in patients receiving driver mutation targeted therapy with tyrosine kinase inhibitors (TKIs) as opposed to patients who did not (27).

\section{Mutations in EGFR}

Therapies against EGFR mutations were the first step towards molecular directed NSCLC therapy. These mutations are mostly seen in exon 19 (deletion) or exon 21 (L858R point mutation) and are detected either in solid tumor biopsies or in liquid biopsies using PCR. They are observed in about $15 \%$ of NSCLC. They are found in $10-20 \%$ of Caucasian patients but in about $48 \%$ of Asian patients with lung cancer (5). Higher incidence of this mutation is also seen with an adenocarcinoma histology, in never smokers, younger patients and in females $(6,7)$. In advanced NSCLC, the presence of EGFR mutation confers a more favorable prognosis. Compared to first line chemotherapy, EGFR TKIs significantly prolonged progression free survival (4.6 to 6.9 months) (8). These include first generation EGFR TKIs (erlotinib, gefitinib), second generation EGFR TKIs (afatinib) and third generation EGFR TKIs (osimertinib).

\section{Translocations in ALK}

This translocation is seen in $1-7 \%$ of $\operatorname{NSCLC~}(9,10)$. It involves an inversion in chromosome 2 that juxtaposes the $5^{\prime}$ end of the echinoderm microtubule-associated protein-like 


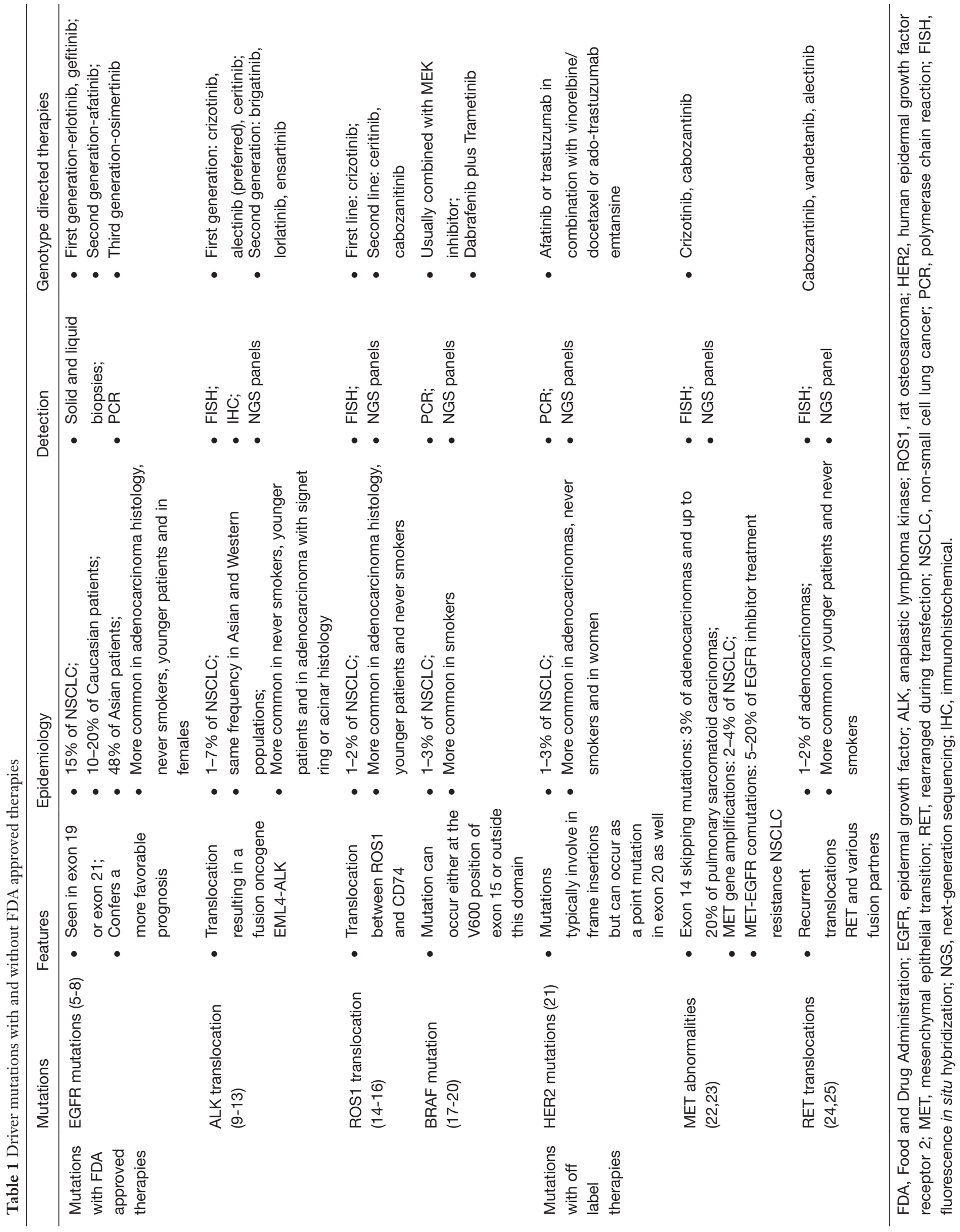


4 (EML4) gene with the 3 ' end of the $A L K$ gene, resulting in the fusion oncogene EML4-ALK. Higher incidence of this translocation is seen in never smokers, younger patients and in adenocarcinoma with signet ring or acinar histology (11). This gene arrangement is mutually exclusive with EGFR and $K R A S$ mutations (11) and is seen in the same frequency in Asian and Western populations (12). ALK translocations can be identified by FISH, IHC or NGS panels. Advanced NSCLCs with $A L K$ fusion oncogene are highly sensitive to ALK TKIs. Crizotinib, a TKI originally developed as a c-MET kinase inhibitor, has shown significant activity in patients with $A L K$ and ROS1 translocation. Compared to first line chemotherapy, Crizotinib significantly prolonged progression free survival (10.9 vs. 7.0 months) (13). Other ALK TKIs include alectinib (now preferred first line) and ceritinib. Second generation ALK TKIs in clinical development, mostly for crizotinib refractory NSCLC, include brigatinib, lorlatinib and ensartinib.

\section{Translocations in ROS1}

ROS 1 translocation, typically between ROS 1 and CD74 (14), is seen in about $1-2 \%$ of NSCLC (15). Higher incidence of this translocation is seen with adenocarcinoma histology, younger patients and never smokers. This translocation can be identified by FISH or by some NGS panels. ROS 1 TK is highly sensitive to crizotinib (response rate of $72 \%$; median progression free survival 19.2 months) (16). Second line agents being studied include ceritinib and cabozanitinib.

\section{Mutations in BRAF}

$B R A F$ is a downstream signaling mediator of $K R A S$ that activates the mitogen-activated protein kinase (MAPK) pathway. This mutation is seen in about $1-3 \%$ of NSCLC and is more common in smokers (17). It is usually detected by PCR or by NGS methods. This mutation can occur either at the V600 position of exon 15 (as in melanoma) or outside this domain. Never or light smokers with this mutation tend to have it more often in the V600 position than the non-V600 position (18). The prognosis is better with the V600 mutation than the non-V600 mutation. Current Food and Drug Administration (FDA) approved therapy for this mutation is usually combination with a MEK inhibitor. In advanced previously treated NSCLC, Dabrafenib plus trametinib was associated with a $63 \%$ response and $79 \%$ disease control rate with a median progression free survival rate of 9.7 (19) to 10.9 months (20).

\section{Other genotypes with off-label therapies}

HER 2 mutations can be detected by PCR or NGS in $1-3 \%$ of NSCLC (21). These mutations typically involve in frame insertions in exon 20 but can occur as a point mutation in exon 20 as well. They are more common in adenocarcinomas, never smokers and in women. For advanced previously treated NSCLC with HER2 exon insertion, afatinib or trastuzumab in combination with vinorelbine/docetaxel or ado-trastuzumab emtansine can be used.

MET abnormalities include exon 14 skipping mutations (3\% of adenocarcinomas and up to $20 \%$ of pulmonary sarcomatoid carcinomas), MET gene amplifications (2-4\% of NSCLC) and MET-EGFR comutations (5-20\% of EGFR inhibitor treatment resistance NSCLC) $(22,23)$. They are detected by NGS panels or by FISH. For advanced previously treated NSCLC, MET inhibitors such as crizotinib and cabozantinib can be used.

Recurrent translocations $R E T$ and various fusion partners, detected by FISH and NGS, have been seen in $1-2 \%$ of adenocarcinomas and typically occur in younger patients and never smokers (24,25). For advanced previously treated NSCLC, cabozantinib, vandetanib and alectinib can be used.

Other genotypes with ongoing clinical trials include $R A S$ mutation (MEK inhibition with trametinib and selumetinib) and PIK3CA, AKT1, PTEN alterations.

\section{Role of ablative bronchoscopic procedures in lung cancer}

Interventional bronchoscopy (Table 2) has an ever-increasing role in the management of advanced lung cancers. The AQuIRE Bronchoscopy Registry looked at 1,115 procedures on 947 patients (66). The technical success for airway reopening was $\sim 93 \%$ with clinically significant relief in dyspnea and improvement in quality of life. Patients with higher dyspnea and lower functional status benefited the most. While the complication rates varied heavily between centers (based on sedation type, use of rigid bronchoscope, type of ventilation and use of stents), the overall rate was still low at about 3.9\% (67).

Multiple factors have influenced the choice of modality 
Table 2 Overview of bronchoscopic ablative procedures

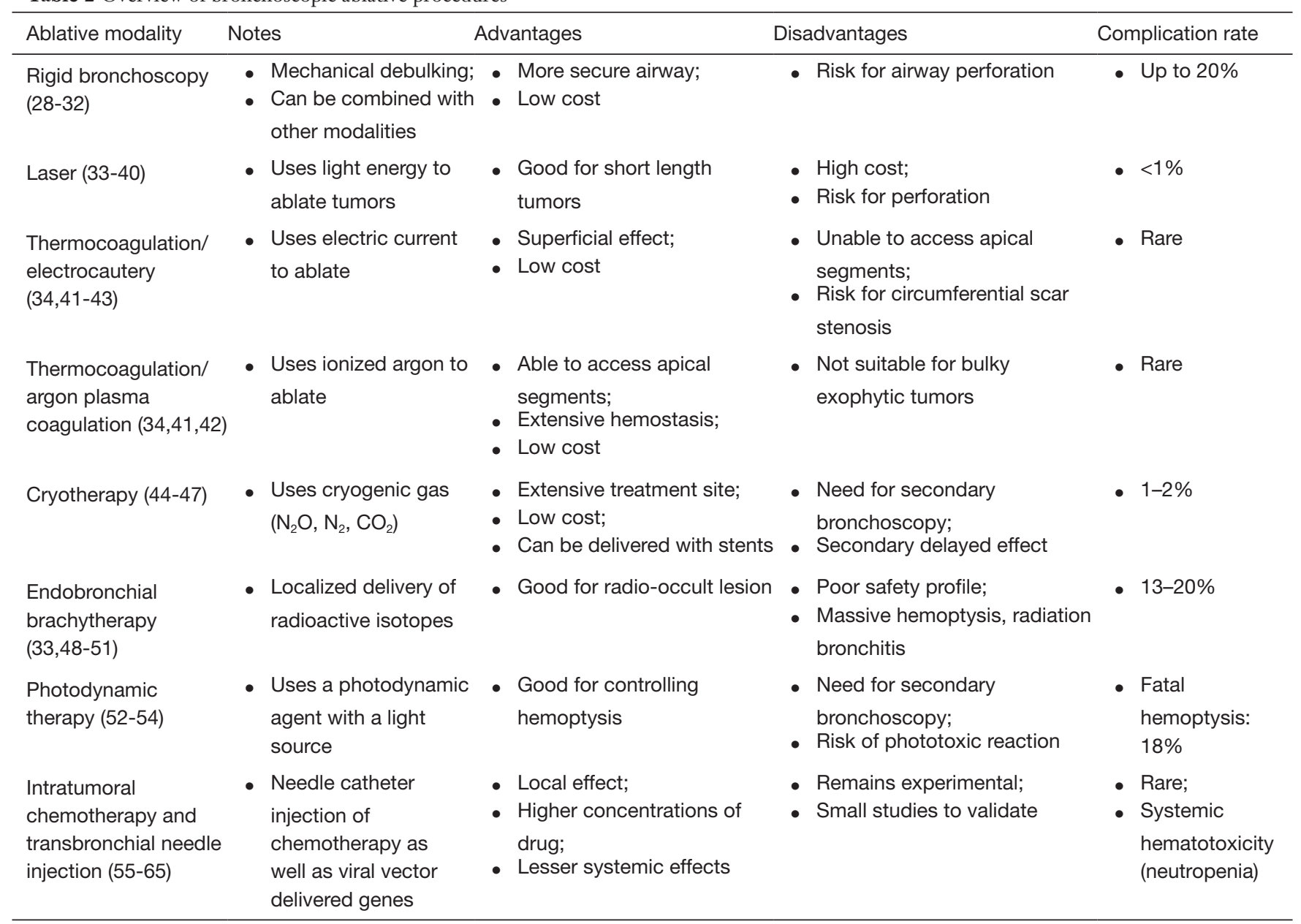

used to manage central airway tumors. These typically involve patient/treatment factors, such as inability to tolerate surgery or "older" generation chemotherapeutic agents. Tumor factors considered usually refer to locations, size and severity of obstruction. The obvious lack of genotype consideration in procedure selection does warrant discussion given advancements made in this area.

\section{Rigid bronchoscopy with mechanical debulking}

The choice between initial flexible vs. rigid bronchoscopy for tumor assessment and management remains controversial. While the complication rates are lower and less serious with flexible bronchoscopy $(<1 \%$ to $6.8 \% v s$. $\sim 20 \%$ ) (28-32), rigid bronchoscopy allows for a more secure airway and possibly safer extended periods for ablative procedures. Older literature supports mechanical debulking with the beveled end alone to "core out" the tumor (airway improvement in $90 \%$ of patients with need for repeat bronchoscopy in $<5 \%$ of patients) (32). In the modern era rigid bronchoscopy is more commonly combined with another ablative or "thermal" modality. As with other procedures, operator experience plays an important role in limiting complications (especially bleeding and airway perforation) while increasing success.

\section{Laser}

'Light amplification by stimulated emission of radiation' uses light energy to ablate (by coagulation or direct destruction) tumors and/or achieve hemostasis by vessel photocoagulation. Laser is mainly indicated for central intraluminal lesions with short length involvement $(<4 \mathrm{~cm})$ and minimal submucosal infiltration $(33,34)$. Laser is often 
and successfully used with rigid bronchoscopy with good restoration of bronchial diameter especially in proximal tumors (trachea and main bronchus) (35). Use of laser in studies has shown improved symptom scores, quality of life, gas exchange and lung function (36-38). Rates of complication, such as severe hemoptysis, and mortality rate associated directly with laser are low $(<1 \%)(38)$. The risk of ignition or airway fire can be prevented by lowering the $\mathrm{FiO}_{2}$ to $<40 \%$. Fatal complications described with laser such as gas embolisms, vascular fistula and mediastinitis $(39,40)$ are very rare. A major limitation with this modality is the cost of the generator and the single-use laser probes.

\section{Thermo-coagulation/electrocautery \& APC}

Electrocautery involves use of high-frequency electric current to deliver thermal energy from the tip of a probe. It can be set for coagulation (at low-power setting, softcoagulation) or vaporization/carbonization (at highpower settings, forced-coagulation) (34). This energy can be delivered by different applicators such as a monopolar probe, bipolar probe, or electric snare, loop, or knife. Electrocautery, unlike laser, has a less deep effect and is ideal for superficial coagulation. While the rate of symptom palliation and lung function improvement observed with electrocautery is similar to laser $(41,42,68)$, the costs of electrocautery are substantially cheaper. Major complications with electrocautery are rare, more so with the soft coagulation mode (43). If applied circumferentially thermocoagulation can cause scarring stenosis (68).

APC is a non-contact form of electrocautery that uses ionized argon as the electrical current. It overcomes 2 limitations noted with electrocautery (I) the ability to access most apical segments (II) improved efficacy in cases of electric current induced bleeding. Argon has the ability to travel upstream in the bloodstream and hence result in hemostasis at the origin of bleeding (41), making it particularly effective in controlling bleeding. As with electrocautery, APC is ideal for superficial coagulation and is mainly indicated for extensive/hemorrhagic tumors prior to mechanical debulking.

In conclusion, both electrocautery and APC are effective low-cost methods of thermocoagulation. They appear to be as effective if not superior to laser in most situations except in bulky exophytic tumors where a deeper coagulation is required.

\section{Cryotherapy}

Cryotherapy uses probes or a spray to deliver cryogenic gases $\left(\mathrm{N}_{2} \mathrm{O}, \mathrm{N}_{2}, \mathrm{CO}_{2}\right)$ at very low temperatures $\left(-196^{\circ} \mathrm{C}\right)$. Cryotherapy has an immediate effect (dehydration and cellular crystallization) and a delayed effect (apoptosis and ischemia) making it an effective short and longer-term modality $(3,44)$. The treatment site is extensive, making cryotherapy ideal for very hemorrhagic tumors where the exact bleeding site is not known. As with other therapies, cryotherapy has been shown to improve airway diameter, performance status and lung function $(45,46)$. Procedure related mortality is low $(\sim 1.2 \%)$ with total complication rates $<10 \%$ (47). As with thermocoagulation, cryotherapy is very cost effective but without the risk of scarring stenosis and with the benefit of an added delayed effect. Finally, cryotherapy can be safely delivered around stents without damaging the prosthesis (4).

\section{$E B T$}

EBT involves deploying radioactive isotopes (iridium 192) in a highly localized manner through a specialized catheter in order to preserve neighboring lung tissue (33). It is used as a sole intervention or in conjunction with external beam radiation either for palliation or curative treatment in selected cases of small tumors $(<3 \mathrm{~cm})(48)$. Despite its potential role in radio-occult lesions and in patients who are poor surgical candidates (contralateral pneumonectomy or respiratory insufficiency), use of EBT is limited by its poor safety profile. Major complications can be seen in $13-20 \%$ of the cases (49). While massive hemoptysis can be seen in up to $7 \%$ of the cases (49), radiation bronchitis with subsequent necrosis and abscess formation can occur in up to $35 \%$ of patients $(50,51)$.

\section{$P D T$}

PDT involves tumor destruction by use of a photosensitizing agent (usually a hematoporphyrin derivative) with a light source $(630 \mathrm{~nm})$. This results in a phototoxic reaction and cell death $(3,33,52)$. PDT typically results in marked, yet delayed, symptom improvement and is not suitable for acute critical proximal obstruction. Repeat bronchoscopy is performed 1-2 days later for removal of necrotic tissue and then 5-7 days later for a second illumination $(52,53)$. PDT is particularly effective in controlling hemoptysis and relieving dyspnea. Lung function improvement is achieved 
in a majority of the cases (53). A phototoxic reaction is noted in $5-28 \%$ of patients and fatal hemoptysis is seen in $\sim 18 \%$ of cases (52). Periprocedural mortalities are usually not treatment related (54).

\section{ITC and TBNI}

While the previously mentioned modalities are mainly directed at acutely managing tumors, ITC and TBNI therapy maybe the first step towards a direct bronchoscopic tumor intervention. Initially, advantages of ITC/TBNI considered included more precision drug delivery, higher tumor drug concentration and less or no systemic toxicity (55). However, emerging evidence suggests that this modality has systemic benefits (and toxicity) and may be used as an adjunct with other interventions such as low dose chemotherapy (56). Needle-catheters used for drug delivery are the same as those used for therapeutic aspiration. In these procedures, chemotherapeutic agents can be injected directly into luminal tumors or into thoracic lymph nodes with the assistance of endobronchial ultrasonography (EBUS). While ITC has been well studied for delivery of chemotherapeutic agents (such as Cisplantin, 5 -Fluorouracil, Bleomycin and Carboplatin) $(57,58)$ it has also been studied for delivery of therapeutic genes via viral vector (59-61).

In an early clinical study (1986-1992) involving 93 patients with inoperable cancer, sequential injection of 5-Fluorouracil, Mitomycin-C, Methotrexate, Bleomycin and Mitoxantrone showed improved airway lumen in $87 \%$ of cases with $>50 \%$ obstruction (57). In another study done in 2000, intratumoral Carboplatin combined with systemic chemotherapy resulted in a better response rate $(90 \% v s$. $55 \%)$, tumor size and dyspnea relief when compared to systemic chemotherapy alone in patients with advanced bronchogenic carcinoma (58). Endobronchial ultrasound guided needle lymph node drug delivery was demonstrated successfully in a study involving 5 patients with EGFR negative advanced bronchogenic cancer who were unfit (performance status 2/PS2) for surgery, radiation and chemotherapy (56). In this proof of concept study, a TBNI protocol using Cisplatin combined with low dose systemic chemotherapy (70\% of standard) was well tolerated by these sick patients with good survival. Since then EBUS-TBNI for drug delivery has been used successfully in patients with severe COPD who had a response enough to tolerate systemic chemotherapy later (62), in patients with local (63) as well as isolated mediastinal/hilar recurrence (64) and also in patients with aspergilloma (65). Studies (BROADWAY, clinicaltrials.gov, NCT02066103) using a new microinfusion device, Blowfish ${ }^{\mathrm{TM}}$ (Mercator MedSystems, Emeryville, CA, USA) are ongoing. Early scientific abstracts suggest that paclitaxel can be delivered safely after rigid bronchoscopy using this specialized catheter in patients with malignant $\mathrm{CAO}$, obviating the need for airway stenting.

While there are no major side effects reported from ITC, systemic effects are noted. In one study patients were reported to have neutropenia $(1,800$ leucocytes per $\mu \mathrm{L})$ after the first intratumoral cisplatin administration (56). Local airway effects appear minimal. Local blanching and decreased tumor bleeding are interesting observations $(55,56)$ that would suggest acute interruption of perfusion or a "shock reaction" of the tumor. Larger experience is needed with this modality for identify other complications.

\section{Other considerations about tumor responsiveness and immunotherapy}

Evolution or change in genotype has been reported before and is a cause of concern for genotype directed therapies (69). The postulations for EGFR mutation change pre and post therapy include the influence of chemotherapy on driver mutations and intratumoral heterogeneity causing a mis-representation of mutational status during initial sampling (70). Smaller nodules appear to have less intratumoral heterogeneity (71) which could explain better long term survival even in the same T-stage descriptor (72). These findings may also be an explanation for the emergence of TKI resistance and treatment failure.

The immune modulatory effects of ablative procedures in cancer is intriguing. It has been well documented with radiofrequency ablation (RFA), cryoablation, microwave ablation therapy, high-intensity focused ultrasound and laser thermotherapy (73). Cryotherapy, for example, is known to increase levels of soluble interleukin-2 receptor, ILD and lymphocyte transformation rate (74). It could also provoke a direct anti-tumor immunity by activating dendritic cells (DCs). Similarly, PDT is known to enhance anti-tumor immunity (75). PDT is known to activate cell mediated $\left(\mathrm{CD} 8^{+} \mathrm{T}\right.$ cell $)$, and to a lesser extent humoral, anti-tumor immunity $(76,77)$. In animal models, PDT ablated mice were able to resist a subsequent tumor challenge (76). In the clinical setting, PDT treated head and neck tumors were noted to have increased immune cell infiltration in distant untreated sites (78) and combing PDT with radical lung sparing pleurectomy for mesothelioma resulted in a less 
aggressive form of recurrence (79); suggesting enhanced tumor immunity. Interestingly, PDT is synergistic with checkpoint inhibitors, such as anti-PD-1/ PD-L1, and results in prolonged anti-tumor immune response $(80,81)$. Similar synergism, where PDT is combined with immunomodulating agents, may also been seen the treatment of other cancers such as vulvar intraepithelial neoplasia(82) and actinic keratosis/ Bowens disease (83) as MHC-I recognition with subsequent $\mathrm{CD}^{+} \mathrm{T}$-cell activation appears to be a key factor for tumor responsiveness. The future of bronchoscopic modalities may also include combinations local tumor RFA with intravenous Paclitaxel and Doxorubicin. Murine model studies have already demonstrated augmented RFA tumor coagulation and endpoint survival with such multi-modality approaches (84). This insight could theoretically translate a multi-step modality. For example, post rigid bronchoscopy debulking local Paclitaxel delivery with the Blowfish ${ }^{\mathrm{TM}}$ (Mercator MedSystems, Emeryville, CA) device could be followed by RFA treatment.

Finally, a professional antigen presenting cell that plays a key role in tumor immunity are dendritic cells (DCs). Immunogenic cell death is characterized by release of damage-associated molecular patterns (DAMPs) (85). DAMPs play a key role in the maturation of DCs. DCs in turn capture, process and present tumor antigens to the appropriate Th1 cells while ultimately leads to the activation of cytotoxic CD8 ${ }^{+} \mathrm{T}$ lymphocytes (86) and hence tumor immunity. Given the tissue damage associated with bronchoscopic ablative modalities, it is quite possible that these procedure result in release in DAMPs, activations and even attraction of DCs to this area and hence development of tumor immunity (87). For example, PDT is known to result the maturation and activation of DCs $(88,89)$. Studies to validate this phenomenon with other modalities are sorely needed. Cisplatin is a systemic immunogenic cell death inducer and in this way a DAMP. Future studies could be centered on its DC maturing effect (hence cancer immunity boosting) via ITC with a staged systemic chemotherapy regimen.

\section{The bronchoscopist in the era of genotype directed lung cancer management}

Referral patterns are shifting, and a change in diagnostic algorithms frequently makes a bronchoscopist the first provider to encounter the patient and the tumor. Reported EBUS-TBNA and EUS-FNA adequacy rates (ranging from $77-98 \%)(90,91)$ almost completely obviates the need for more invasive surgical tumor sampling. In fact, EBUSTBNA has one of the lowest insufficiency rates (4\%) for EGFR and KRAS mutation analysis; compared to CT guided needle aspiration (7.5\%) and ultrasound guided superficial fine needle aspiration (10\%) (91). Given that $\sim 25 \%$ of patients with enlarging lymph nodes following curative NSCLC surgery have a second primary lung cancer (92), a bronchoscopist also plays a role in the long term follow-up/restaging of these patients. This is also important given the emergence of acquired resistance to the newer TKIs.

The cause of acquired resistance is not fully understood. It is proposed that resistance to EGFR TKIs could be the result of a secondary mutation in EGFR (most commonly substitution of methionine for threonine at position 790 i.e., T790M) $(93,94)$ or the amplification of the MET oncogene (which can occur with or without the T790M mutation) (24). Regardless of the mechanism, a bronchoscopist may be required to resample the tumor at this point when the patients are frequently very sick. Insight into driver mutations while deciding on the optimal therapeutic modality is analogous, in some ways, to knowledge on TNM staging while EBUS-TBNA lymph node staging.

Another evolving concept in advanced NSCLC is the abscopal response noted with combination of radiation and immunotherapy (95). This rare phenomenon, wherein distant lesions regress after radiotherapy to a targeted site has been, has been described for many years now (96). In one study, $25 \%$ of melanoma patients showed regression of non-irradiated lesions when immunotherapy was continued after radiation to the tumor site after it had failed immunotherapy alone (anti-PD-1 monotherapy) (97). It was first reported in 2013 in a patient with treatment refractory lung cancer treated with radiotherapy and Ipilimumab (anti-CTL4-monoclonal antibody) (95). The concept of "boosting" immune responses to treat remote lesions opens another avenue for the bronchoscopist. It would be intriguing to explore whether such a response is also noted (or exaggerated) based on the ablative modality used (for example: EBT vs. PDT vs. APC).

There is a plethora of questions that remain unanswered. What is the effect of various bronchoscopic ablative procedures on tissue sampling for advanced previously treated NSCLC? Does the procedure itself have any effect of driver mutation either in vivo or ex vitro? Should driver mutations influence our choice of modality? Is there an abscopal response noted with different modalities as noted 
with radiotherapy combined with immunotherapy?

\section{Conclusion/future direction}

Ground breaking advances in our understanding of driver mutations of lung cancer and in the technology available for bronchoscopic ablation have completely changed the landscape of advanced lung cancer management. As the two fields progress in parallel, we must ask ourselves if they should be bought together in the name of precision medicine. We must also wonder if genotypes of cancer produce specific endotypes and whether these endotypes respond differently to each bronchoscopic modality.

\section{Acknowledgements}

None.

\section{Footnote}

Conflicts of Interest: MR Bowling is a Consultant for Medtronic, Biodesix and Veracyte. W Hohenforst-Schmidt is a Consultant for Medtronic and Philips. Other authors have no conflicts of interest to declare.

\section{References}

1. Torre LA, Bray F, Siegel RL, et al. Global cancer statistics, 2012. CA Cancer J Clin 2015;65:87-108.

2. Ferlay J, Soerjomataram I, Dikshit R, et al. Cancer incidence and mortality worldwide: sources, methods and major patterns in GLOBOCAN 2012. Int J Cancer 2015;136:E359-86.

3. Ernst A, Feller-Kopman D, Becker HD, et al. Central airway obstruction. Am J Respir Crit Care Med 2004;169:1278-97.

4. Guibert N, Mazieres J, Marquette CH, et al. Integration of interventional bronchoscopy in the management of lung cancer. Eur Respir Rev 2015;24:378-91.

5. Dearden S, Stevens J, Wu YL, et al. Mutation incidence and coincidence in non small-cell lung cancer: metaanalyses by ethnicity and histology (mutMap). Ann Oncol 2013;24:2371-6.

6. Shigematsu H, Lin L, Takahashi T, et al. Clinical and biological features associated with epidermal growth factor receptor gene mutations in lung cancers. J Natl Cancer Inst 2005;97:339-46.

7. Kawaguchi T, Koh Y, Ando M, et al. Prospective Analysis of Oncogenic Driver Mutations and Environmental Factors: Japan Molecular Epidemiology for Lung Cancer Study. J Clin Oncol 2016;34:2247-57.

8. Lee CK, Wu YL, Ding PN, et al. Impact of Specific Epidermal Growth Factor Receptor (EGFR) Mutations and Clinical Characteristics on Outcomes After Treatment With EGFR Tyrosine Kinase Inhibitors Versus Chemotherapy in EGFR-Mutant Lung Cancer: A MetaAnalysis. J Clin Oncol 2015;33:1958-65.

9. Kwak EL, Bang YJ, Camidge DR, et al. Anaplastic lymphoma kinase inhibition in non-small-cell lung cancer. N Engl J Med 2010;363:1693-703.

10. Pikor LA, Ramnarine VR, Lam S, et al. Genetic alterations defining NSCLC subtypes and their therapeutic implications. Lung Cancer 2013;82:179-89.

11. Takahashi T, Sonobe M, Kobayashi M, et al. Clinicopathologic features of non-small-cell lung cancer with EML4-ALK fusion gene. Ann Surg Oncol 2010;17:889-97.

12. Solomon B, Varella-Garcia M, Camidge DR. ALK gene rearrangements: a new therapeutic target in a molecularly defined subset of non-small cell lung cancer. J Thorac Oncol 2009;4:1450-4.

13. Solomon BJ, Mok T, Kim DW, et al. First-line crizotinib versus chemotherapy in ALK-positive lung cancer. $\mathrm{N}$ Engl J Med 2014;371:2167-77.

14. Bergethon K, Shaw AT, Ou SH, et al. ROS1 rearrangements define a unique molecular class of lung cancers. J Clin Oncol 2012;30:863-70.

15. Rimkunas VM, Crosby KE, Li D, et al. Analysis of receptor tyrosine kinase ROS1-positive tumors in nonsmall cell lung cancer: identification of a FIG-ROS1 fusion. Clin Cancer Res 2012;18:4449-57.

16. Shaw AT, Ou SH, Bang YJ, et al. Crizotinib in ROS1rearranged non-small-cell lung cancer. $\mathrm{N}$ Engl J Med 2014;371:1963-71.

17. Paik PK, Arcila ME, Fara M, et al. Clinical characteristics of patients with lung adenocarcinomas harboring BRAF mutations. J Clin Oncol 2011;29:2046-51.

18. Litvak AM, Paik PK, Woo KM, et al. Clinical characteristics and course of 63 patients with BRAF mutant lung cancers. J Thorac Oncol 2014;9:1669-74.

19. Planchard D, Besse B, Groen HJM, et al. Dabrafenib plus trametinib in patients with previously treated BRAF(V600E)-mutant metastatic non-small cell lung cancer: an open-label, multicentre phase 2 trial. Lancet Oncol 2016;17:984-93.

20. Planchard D, Smit EF, Groen HJM, et al. Dabrafenib 
plus trametinib in patients with previously untreated BRAF(V600E)-mutant metastatic non-small-cell lung cancer: an open-label, phase 2 trial. Lancet Oncol 2017;18:1307-16.

21. Pillai RN, Behera M, Berry LD, et al. HER2 mutations in lung adenocarcinomas: A report from the Lung Cancer Mutation Consortium. Cancer 2017;123:4099-105.

22. Bean J, Brennan C, Shih JY, et al. MET amplification occurs with or without T790M mutations in EGFR mutant lung tumors with acquired resistance to gefitinib or erlotinib. Proc Natl Acad Sci U S A 2007;104:20932-7.

23. Kubo T, Yamamoto H, Lockwood WW, et al. MET gene amplification or EGFR mutation activate MET in lung cancers untreated with EGFR tyrosine kinase inhibitors. Int J Cancer 2009;124:1778-84.

24. Takeuchi K, Soda M, Togashi Y, et al. RET, ROS1 and ALK fusions in lung cancer. Nat Med 2012;18:378-81.

25. Wang $\mathrm{R}, \mathrm{Hu} \mathrm{H}, \mathrm{Pan} \mathrm{Y}$, et al. RET fusions define a unique molecular and clinicopathologic subtype of non-small-cell lung cancer. J Clin Oncol 2012;30:4352-9.

26. Kris MG, Johnson BE, Berry LD, et al. Using multiplexed assays of oncogenic drivers in lung cancers to select targeted drugs. JAMA 2014;311:1998-2006.

27. Aurora RN, Casey KR, Kristo D, et al. Practice parameters for the surgical modifications of the upper airway for obstructive sleep apnea in adults. Sleep 2010;33:1408-13.

28. Ost DE, Ernst A, Lei X, et al. Diagnostic Yield and Complications of Bronchoscopy for Peripheral Lung Lesions. Results of the AQuIRE Registry. Am J Respir Crit Care Med 2016;193:68-77.

29. Facciolongo N, Patelli M, Gasparini S, et al. Incidence of complications in bronchoscopy. Multicentre prospective study of 20,986 bronchoscopies. Monaldi Arch Chest Dis 2009;71:8-14.

30. Jin F, Mu D, Chu D, et al. Severe complications of bronchoscopy. Respiration 2008;76:429-33.

31. Pue CA, Pacht ER. Complications of fiberoptic bronchoscopy at a university hospital. Chest 1995;107:430-2.

32. Mathisen DJ, Grillo HC. Endoscopic relief of malignant airway obstruction. Ann Thorac Surg 1989;48:469-73; discussion 473-5.

33. Bolliger CT, Mathur PN, Beamis JF, et al. ERS/ATS statement on interventional pulmonology. European Respiratory Society/American Thoracic Society. Eur Respir J 2002;19:356-73.

34. Ernst A, Silvestri GA, Johnstone D, et al. Interventional pulmonary procedures: Guidelines from the American
College of Chest Physicians. Chest 2003;123:1693-717.

35. Cavaliere S, Foccoli P, Farina PL. Nd:YAG laser bronchoscopy. A five-year experience with 1,396 applications in 1,000 patients. Chest 1988;94:15-21.

36. Kvale PA, Eichenhorn MS, Radke JR, et al. YAG laser photoresection of lesions obstructing the central airways. Chest 1985;87:283-8.

37. Han CC, Prasetyo D, Wright GM. Endobronchial palliation using Nd:YAG laser is associated with improved survival when combined with multimodal adjuvant treatments. J Thorac Oncol 2007;2:59-64.

38. Venuta F, Rendina EA, De Giacomo T, et al. Nd:YAG laser resection of lung cancer invading the airway as a bridge to surgery and palliative treatment. Ann Thorac Surg 2002;74:995-8.

39. Dumon JF, Shapshay S, Bourcereau J, et al. Principles for safety in application of neodymium-YAG laser in bronchology. Chest 1984;86:163-8.

40. Brutinel WM, Cortese DA, McDougall JC, et al. A two-year experience with the neodymium-YAG laser in endobronchial obstruction. Chest 1987;91:159-65.

41. Tremblay A, Marquette CH. Endobronchial electrocautery and argon plasma coagulation: a practical approach. Can Respir J 2004;11:305-10.

42. Petrou M, Kaplan D, Goldstraw P. Bronchoscopic diathermy resection and stent insertion: a cost effective treatment for tracheobronchial obstruction. Thorax 1993;48:1156-9.

43. Hooper RG, Jackson FN. Endobronchial electrocautery. Chest 1988;94:595-8.

44. Marasso A, Gallo E, Massaglia GM, et al. Cryosurgery in bronchoscopic treatment of tracheobronchial stenosis. Indications, limits, personal experience. Chest 1993;103:472-4.

45. Hetzel M, Hetzel J, Schumann C, et al. Cryorecanalization: a new approach for the immediate management of acute airway obstruction. J Thorac Cardiovasc Surg 2004;127:1427-31.

46. Maiwand MO. The role of cryosurgery in palliation of tracheo-bronchial carcinoma. Eur J Cardiothorac Surg 1999;15:764-8.

47. Maiwand MO, Asimakopoulos G. Cryosurgery for lung cancer: clinical results and technical aspects. Technol Cancer Res Treat 2004;3:143-50.

48. Skowronek J. Brachytherapy in the treatment of lung cancer - a valuable solution. J Contemp Brachytherapy 2015;7:297-311.

49. Speiser BL, Spratling L. Remote afterloading 
brachytherapy for the local control of endobronchial carcinoma. Int J Radiat Oncol Biol Phys 1993;25:579-87.

50. Huber RM, Fischer R, Hautmann H, et al. Palliative endobronchial brachytherapy for central lung tumors. A prospective, randomized comparison of two fractionation schedules. Chest 1995;107:463-70.

51. Tredaniel J, Hennequin C, Zalcman G, et al. Prolonged survival after high-dose rate endobronchial radiation for malignant airway obstruction. Chest 1994;105:767-72.

52. Moghissi K, Dixon K, Thorpe JA, et al. Photodynamic therapy (PDT) in early central lung cancer: a treatment option for patients ineligible for surgical resection. Thorax 2007;62:391-5.

53. Moghissi K, Dixon K, Stringer M, et al. The place of bronchoscopic photodynamic therapy in advanced unresectable lung cancer: experience of 100 cases. Eur J Cardiothorac Surg 1999;15:1-6.

54. Minnich DJ, Bryant AS, Dooley A, et al. Photodynamic laser therapy for lesions in the airway. Ann Thorac Surg 2010;89:1744-8; discussion 8-9.

55. Celikoglu F, Celikoglu SI, Goldberg EP. Bronchoscopic intratumoral chemotherapy of lung cancer. Lung Cancer 2008;61:1-12.

56. Hohenforst-Schmidt W, Zarogoulidis P, Darwiche K, et al. Intratumoral chemotherapy for lung cancer: rechallenge current targeted therapies. Drug Des Devel Ther 2013;7:571-83.

57. Celikoglu SI, Karayel T, Demirci S, et al. Direct injection of anti-cancer drugs into endobronchial tumours for palliation of major airway obstruction. Postgrad Med J 1997;73:159-62.

58. Liu M, Ma P, Lu Z. [Local chemotherapy by fibrobronchoscopy for advanced bronchogenic carcinoma]. Zhonghua Jie He He Hu Xi Za Zhi 2000;23:550-1.

59. Swisher SG, Roth JA, Nemunaitis J, et al. Adenovirusmediated $\mathrm{p} 53$ gene transfer in advanced non-small-cell lung cancer. J Natl Cancer Inst 1999;91:763-71.

60. Schuler M, Herrmann R, De Greve JL, et al. Adenovirusmediated wild-type $\mathrm{p} 53$ gene transfer in patients receiving chemotherapy for advanced non-small-cell lung cancer: results of a multicenter phase II study. J Clin Oncol 2001;19:1750-8.

61. Griscelli F, Opolon P, Saulnier P, et al. Recombinant adenovirus shedding after intratumoral gene transfer in lung cancer patients. Gene Ther 2003;10:386-95.

62. Li X, Liu X, Rao X, et al. A case report of local treatment of inoperable squamous cell lung carcinoma with convexprobe endobronchial ultrasound-guided intratumoral injection of cisplatin in a patient with severe COPD. Medicine (Baltimore) 2017;96:e7070.

63. Khan F, Anker CJ, Garrison G, et al. Endobronchial ultrasound-guided transbronchial needle injection for local control of recurrent non-small cell lung cancer. Ann Am Thorac Soc 2015;12:101-4.

64. Mehta HJ, Begnaud A, Penley AM, et al. Treatment of isolated mediastinal and hilar recurrence of lung cancer with bronchoscopic endobronchial ultrasound guided intratumoral injection of chemotherapy with cisplatin. Lung Cancer 2015;90:542-7.

65. Parikh MS, Seeley E, Nguyen-Tran E, et al. Endobronchial Ultrasound-guided Transbronchial Needle Injection of Liposomal Amphotericin B for the Treatment of Symptomatic Aspergilloma. J Bronchology Interv Pulmonol 2017;24:330-3.

66. Ost DE, Ernst A, Grosu HB, et al. Therapeutic bronchoscopy for malignant central airway obstruction: success rates and impact on dyspnea and quality of life. Chest 2015;147:1282-98.

67. Ost DE, Ernst A, Grosu HB, et al. Complications Following Therapeutic Bronchoscopy for Malignant Central Airway Obstruction: Results of the AQuIRE Registry. Chest 2015;148:450-71.

68. Coulter TD, Mehta AC. The heat is on: impact of endobronchial electrosurgery on the need for Nd-YAG laser photoresection. Chest 2000;118:516-21.

69. Bai H, Wang Z, Chen K, et al. Influence of chemotherapy on EGFR mutation status among patients with non-smallcell lung cancer. J Clin Oncol 2012;30:3077-83.

70. Luo YH, Chen YM. Influence of chemotherapy on EGFR mutation status. Transl Lung Cancer Res 2013;2:442-4.

71. Lipinski KA, Barber LJ, Davies MN, et al. Cancer Evolution and the Limits of Predictability in Precision Cancer Medicine. Trends Cancer 2016;2:49-63.

72. Takenaka T, Yamazaki K, Miura N, et al. The Prognostic Impact of Tumor Volume in Patients with Clinical Stage IA Non-Small Cell Lung Cancer. J Thorac Oncol 2016;11:1074-80.

73. Haen SP, Pereira PL, Salih HR, et al. More than just tumor destruction: immunomodulation by thermal ablation of cancer. Clin Dev Immunol 2011;2011:160250.

74. Zhou P, Lu Z, Zhang G. A study on immunity enhancement of frozen human lung cancer cells in vitro. Zhongguo Fei Ai Za Zhi 1999;2:32-4.

75. Shafirstein G, Battoo A, Harris K, et al. Photodynamic Therapy of Non-Small Cell Lung Cancer. Narrative Review and Future Directions. Ann Am Thorac Soc 
2016;13:265-75.

76. Korbelik M, Krosl G, Krosl J, et al. The role of host lymphoid populations in the response of mouse EMT6 tumor to photodynamic therapy. Cancer Res 1996;56:5647-52.

77. Preise D, Oren R, Glinert I, et al. Systemic antitumor protection by vascular-targeted photodynamic therapy involves cellular and humoral immunity. Cancer Immunol Immunother 2009;58:71-84.

78. Thong PS, Ong KW, Goh NS, et al. Photodynamictherapy-activated immune response against distant untreated tumours in recurrent angiosarcoma. Lancet Oncol 2007;8:950-2.

79. Friedberg JS. Radical pleurectomy and photodynamic therapy for malignant pleural mesothelioma. Ann Cardiothorac Surg 2012;1:472-80.

80. He C, Duan X, Guo N, et al. Core-shell nanoscale coordination polymers combine chemotherapy and photodynamic therapy to potentiate checkpoint blockade cancer immunotherapy. Nat Commun 2016;7:12499.

81. Gao L, Zhang C, Gao D, et al. Enhanced Anti-Tumor Efficacy through a Combination of Integrin alphavbeta6Targeted Photodynamic Therapy and Immune Checkpoint Inhibition. Theranostics 2016;6:627-37.

82. Maeurer MJ, Gollin SM, Storkus WJ, et al. Tumor escape from immune recognition: loss of HLA-A2 melanoma cell surface expression is associated with a complex rearrangement of the short arm of chromosome 6. Clin Cancer Res 1996;2:641-52.

83. Dragieva G, Hafner J, Dummer R, et al. Topical photodynamic therapy in the treatment of actinic keratoses and Bowen's disease in transplant recipients. Transplantation 2004;77:115-21.

84. Yang W, Ahmed M, Elian M, et al. Do liposomal apoptotic enhancers increase tumor coagulation and end-point survival in percutaneous radiofrequency ablation of tumors in a rat tumor model? Radiology 2010;257:685-96.

85. Krysko O, Love Aaes T, Bachert C, et al. Many faces of DAMPs in cancer therapy. Cell Death Dis 2013;4:e631.

86. Nace G, Evankovich J, Eid R, et al. Dendritic cells and damage-associated molecular patterns: endogenous danger signals linking innate and adaptive immunity. J Innate Immun 2012;4:6-15.

87. Keisari Y. Tumor abolition and antitumor immunostimulation by physico-chemical tumor ablation. Front Biosci (Landmark Ed) 2017;22:310-47.

88. Gollnick SO, Owczarczak B, Maier P. Photodynamic therapy and anti-tumor immunity. Lasers Surg Med 2006;38:509-15.

89. Sur BW, Nguyen P, Sun CH, et al. Immunophototherapy using PDT combined with rapid intratumoral dendritic cell injection. Photochem Photobiol 2008;84:1257-64.

90. Nakajima T, Yasufuku K, Suzuki M, et al. Assessment of epidermal growth factor receptor mutation by endobronchial ultrasound-guided transbronchial needle aspiration. Chest 2007;132:597-602.

91. Billah S, Stewart J, Staerkel G, et al. EGFR and KRAS mutations in lung carcinoma: molecular testing by using cytology specimens. Cancer Cytopathol 2011;119:111-7.

92. Anraku M, Pierre AF, Nakajima T, et al. Endobronchial ultrasound-guided transbronchial needle aspiration in the management of previously treated lung cancer. Ann Thorac Surg 2011;92:251-5; discussion 5.

93. Balak MN, Gong Y, Riely GJ, et al. Novel D761Y and common secondary T790M mutations in epidermal growth factor receptor-mutant lung adenocarcinomas with acquired resistance to kinase inhibitors. Clin Cancer Res 2006;12:6494-501.

94. Kobayashi S, Boggon TJ, Dayaram T, et al. EGFR mutation and resistance of non-small-cell lung cancer to gefitinib. N Engl J Med 2005;352:786-92.

95. Golden EB, Demaria S, Schiff PB, et al. An abscopal response to radiation and ipilimumab in a patient with metastatic non-small cell lung cancer. Cancer Immunol Res 2013;1:365-72.

96. Mole RH. Whole body irradiation; radiobiology or medicine? Br J Radiol 1953;26:234-41.

97. Ribeiro Gomes J, Schmerling RA, Haddad CK, et al. Analysis of the Abscopal Effect With Anti-PD1 Therapy in Patients With Metastatic Solid Tumors. J Immunother 2016;39:367-72.
Cite this article as: Mohan A, Harris K, Bowling MR, Brown C, Hohenforst-Schmidt W. Therapeutic bronchoscopy in the era of genotype directed lung cancer management. J Thorac Dis 2018;10(11):6298-6309. doi: 10.21037/jtd.2018.08.14 